\title{
Heisenberg uniqueness pairs for the hyperbola
}

\author{
Deb Kumar Giri and Rama Rawat
}

\begin{abstract}
Let $\Gamma$ be the hyperbola $\left\{(x, y) \in \mathbb{R}^{2}: x y=1\right\}$ and $\Lambda_{\beta}$ be the lattice-cross defined by $\Lambda_{\beta}=(\mathbb{Z} \times$ $\{0\}) \cup(\{0\} \times \beta \mathbb{Z})$ in $\mathbb{R}^{2}$, where $\beta$ is a positive real. A result of Hedenmalm and Montes-Rodríguez says that $\left(\Gamma, \Lambda_{\beta}\right)$ is a Heisenberg uniqueness pair if and only if $\beta \leqslant 1$. In this paper, we show that for a rational perturbation of $\Lambda_{\beta}$, namely

$$
\Lambda_{\beta}^{\theta}=((\mathbb{Z}+\{\theta\}) \times\{0\}) \cup(\{0\} \times \beta \mathbb{Z}),
$$

where $\theta=1 / p$, for some $p \in \mathbb{N}$ and $\beta$ is a positive real, the pair $\left(\Gamma, \Lambda_{\beta}^{\theta}\right)$ is a Heisenberg uniqueness pair if and only if $\beta \leqslant p$.
\end{abstract}

\section{Introduction}

The notion of Heisenberg uniqueness pair has been around for some time now. It was first introduced in [12] by Hedenmalm and Montes-Rodríguez as a variant of the uncertainty principle for Fourier transform, which says that a nonzero function and its Fourier transform cannot be simultaneously too small (for more on uncertainty principle see $[\mathbf{3}, \mathbf{1 1}, \mathbf{1 5}]$ ). In $[\mathbf{1 2}]$, authors had proposed the following. Let $\Gamma$ be a finite disjoint union of smooth curves in $\mathbb{R}^{2}$ and $\Lambda$ be any subset of $\mathbb{R}^{2}$. Let $X(\Gamma)$ be the space of all finite complex-valued Borel measures $\mu$ in $\mathbb{R}^{2}$ which are supported on $\Gamma$ and are absolutely continuous with respect to the arc length measure on $\Gamma$. For $(\xi, \eta) \in \mathbb{R}^{2}$, the Fourier transform of $\mu$ is defined by

$$
\hat{\mu}(\xi, \eta)=\int_{\Gamma} e^{\pi i(x \xi+y \eta)} d \mu(x, y) .
$$

Definition 1.1. A pair $(\Gamma, \Lambda)$ is said to be a Heisenberg uniqueness pair (HUP), if the only $\mu \in X(\Gamma)$ satisfying $\hat{\mu}(\xi, \eta)=0$ for all $(\xi, \eta) \in \Lambda$, is the zero measure.

Thus if $(\Gamma, \Lambda)$ is a HUP, then the set $\Lambda$ determines $\mu \in X(\Gamma)$. In interesting examples of HUP, the set $\Lambda$, for a given $\Gamma$, is taken to be a very 'small' subset of Euclidean plane. This constraint on $\Lambda$ (for a given $\Gamma$ ) seems to make Heisenberg uniqueness pairs to be on the other extreme of uncertainty principles and makes investigating them a challenging problem.

The expression (1.1) of Fourier transform is not the standard one, but since the problem we take up here is inspired by and closely follows the methods of [12], we will follow definitions and notation from [12] as far as possible. Moreover, the definition of Heisenberg uniqueness pair can be and in fact has been extended to more general settings as in the work [9, 16-20], where several examples of Heisenberg uniqueness pairs have been obtained.

Invariance properties. The properties of Fourier transform together with change of variables imply the following invariance properties for the Heisenberg uniqueness pair.

Received 3 January 2020; revised 25 May 2020; published online 16 July 2020.

2010 Mathematics Subject Classification 42A10, 42B10 (primary), 37A45 (secondary).

The first author was supported by Institute Post-doctoral Fellowship, Indian Institute of Technology Kanpur, India.

(C) 2020 The Authors. The publishing rights in this article are licensed to the London Mathematical Society under an exclusive licence. 
(i) Let $u, v \in \mathbb{R}^{2}$. The pair $(\Gamma, \Lambda)$ is a HUP if and only if the pair $(\Gamma+\{u\}, \Lambda+\{v\})$ is a HUP.

(ii) Let $T: \mathbb{R}^{2} \rightarrow \mathbb{R}^{2}$ be an invertible linear transform and $T^{*}$ be its adjoint. The pair $(\Gamma, \Lambda)$ is a HUP if and only if the pair $\left(T^{-1} \Gamma, T^{*} \Lambda\right)$ is a HUP.

Dual condition for HUP. For $\zeta \equiv(\xi, \eta) \in \Lambda$, define a function $e_{\zeta}$ on $\Gamma$ by $e_{\zeta}(x, y)=e^{\pi i(x \xi+y \eta)}$. Then the pair $(\Gamma, \Lambda)$ is a HUP if and only if the set $\left\{e_{\zeta}: \zeta \in \Lambda\right\}$ spans a weak-star dense subspace of $L^{\infty}(\Gamma)$.

Our focus on this paper is on a result from [12], where authors have studied the pair (hyperbola, some lattice-cross) and have proved the following result.

Theorem $1.2[\mathbf{1 2}]$. Let $\Gamma=\left\{(x, y) \in \mathbb{R}^{2}: x y=1\right\}$ be the hyperbola and $\Lambda_{\beta}$ be the latticecross $\Lambda_{\beta}=(\mathbb{Z} \times\{0\}) \cup(\{0\} \times \beta \mathbb{Z})$, where $\beta$ is a positive real. Then $\left(\Gamma, \Lambda_{\beta}\right)$ is a Heisenberg uniqueness pair if and only if $\beta \leqslant 1$.

By duality, Theorem 1.2 is equivalent to the following density result.

TheOrem $1.3[\mathbf{1 2}]$. The space of all linear span of the functions $\left\{e_{n}(x)=e^{\pi i n x} ; n \in \mathbb{Z}\right\} \cup$ $\left\{e_{n}^{\langle\beta\rangle}(x)=e^{\pi i n \beta / x} ; n \in \mathbb{Z}\right\}$ is weak-star dense in $L^{\infty}(\mathbb{R})$ if and only if $0<\beta \leqslant 1$.

Many examples of Heisenberg uniqueness pair have been obtained in the setting of the plane as well as in the higher dimensional Euclidean spaces. For more details see $[\mathbf{1}, \mathbf{2}, \mathbf{5}, \mathbf{7 - 1 0}, \mathbf{1 3}$, 14, 16-20].

Next, we state the main result of this paper which is a variant of Theorem 1.2 and the concerned problem finds a mention in (Section 7, open problem $(a),[\mathbf{1 2}]$ ).

Theorem 1.4. Let $\Gamma=\left\{(x, y) \in \mathbb{R}^{2}: x y=1\right\}$ be the hyperbola and $\Lambda_{\beta}^{\theta}$ be the latticecross

$$
\Lambda_{\beta}^{\theta}=((\mathbb{Z}+\{\theta\}) \times\{0\}) \cup(\{0\} \times \beta \mathbb{Z}),
$$

where $\theta=1 / p$, for some $p \in \mathbb{N}$ and $\beta$ is a positive real. Then $\left(\Gamma, \Lambda_{\beta}^{\theta}\right)$ is a Heisenberg uniqueness pair if and only if $\beta \leqslant p$.

Corollary 1.5. Let $\Gamma=\left\{(x, y) \in \mathbb{R}^{2}: x y=1\right\}$ be the hyperbola and $\Lambda_{\beta}^{\theta}$ be the set $\Lambda_{\beta}^{\theta}=((\mathbb{Z}+\{\theta\}) \times\{0\}) \cup(\{0\} \times \beta \mathbb{Z})$, where $\theta=q / p$, for some $p \in \mathbb{N}$ and $q \in \mathbb{Z}$ with $\operatorname{gcd}(\mathrm{p}, \mathrm{q})=1$ and $\beta$ is a positive real. Then $\left(\Gamma, \Lambda_{\beta}^{\theta}\right)$ is a Heisenberg uniqueness pair if and only if $\beta \leqslant p$.

REMARK 1.6. (i) The definition of HUP, may be extended to include more general measures $\mu$, than only those which are absolutely continuous with respect to the arc length measure on $\Gamma$. But for our purpose, it is essential that the measure $\mu$ is absolutely continuous with respect to the arc length measure, for without it Theorem 1.4 is not true. To see this, we consider the singular measure $\mu=\delta_{u}-\delta_{v}$ on $\Gamma=\left\{(x, y) \in \mathbb{R}^{2}: x y=1\right\}$, where $\delta_{s}$ denotes the point mass at $s \in \Gamma$ and $u=\left(u_{0}, 1 / u_{0}\right) \in \Gamma, v=\left(v_{0}, 1 / v_{0}\right) \in \Gamma$. Then it is clear from (1.1) that

$$
\hat{\mu}(\xi, 0)=e^{\pi i \xi u_{0}}-e^{\pi i \xi v_{0}} \text { and } \hat{\mu}(0, \eta)=e^{\pi i \eta / u_{0}}-e^{\pi i \eta / v_{0}} .
$$

A simple computation shows that there are many different points like $u_{0}, v_{0} \in \mathbb{R} \backslash\{0\}$ such that $\left.\hat{\mu}\right|_{\Lambda_{\beta}^{\theta}}=0$. 
(ii) The presence of $\theta$ showing up in the condition of $\beta$ is somewhat unexpected. The proof of Theorem 1.4 is a careful adaptation of methods in [12]. The question is still open when $\theta$ is irrational.

(iii) For any polynomial $P$ in $\mathbb{R}^{2}$, the Fourier transform of $\mu$ satisfies

$$
P\left(\frac{\partial_{\xi}}{i \pi}, \frac{\partial_{\eta}}{i \pi}\right) \hat{\mu}(\xi, \eta)=\int_{\mathbb{R}^{2}} e^{i \pi(x \xi+y \eta)} P(x, y) d \mu(x, y)
$$

in the sense of distributions. In particular, if $P(x, y):=x y-1$ and $\Gamma$ is the zero set of $P$, that is, the hyperbola $x y=1$, then any $\mu \in X(\Gamma), u:=\hat{\mu}$ is a solution of the one-dimensional Klein-Gordon equation

$$
\left(\partial_{\xi} \partial_{\eta}+\pi^{2}\right) u(\xi, \eta)=0 .
$$

Therefore, $(\Gamma, \Lambda)$ is a HUP if and only if the above partial differential equation, with the initial condition $u=0$ on $\Lambda$ has only a trivial solution.

(iv) Let $\mathcal{A C}(\Gamma, \Lambda)=\left\{\mu \in X(\Gamma):\left.\widehat{\mu}\right|_{\Lambda}=0\right\}$. Let $\Gamma, \Lambda_{\beta}^{\theta}$ be as in the Theorem 1.4. Then $\mathcal{A C}\left(\Gamma, \Lambda_{\beta}^{\theta}\right)=\{0\}$ if and only if $\beta \leqslant p$. For $p<\beta<\infty$, it seems likely that $\mathcal{A C}\left(\Gamma, \Lambda_{\beta}^{\theta}\right)$ is infinite-dimensional in analogy with the results in [4]. This question is still open.

\section{Proof of the main result}

Let $\Gamma=\left\{(x, y) \in \mathbb{R}^{2}: \quad x y=1\right\}$ be the hyperbola and $\mu \in X(\Gamma)$, then there exists $f \in L^{1}\left(\mathbb{R}, \sqrt{1+1 / t^{4}} d t\right)$ such that for bounded and continuous function $\varphi$ on $\mathbb{R}^{2}$,

$$
\int_{\Gamma} \varphi(x, y) d \mu(x, y)=\int_{\mathbb{R} \backslash\{0\}} \varphi(t, 1 / t) f(t) \sqrt{1+1 / t^{4}} d t .
$$

In particular, the Fourier transform of $\mu$ can be expressed as

$$
\hat{\mu}\left(\xi_{1}, \xi_{2}\right)=\int_{\mathbb{R} \backslash\{0\}} e^{\pi i\left(\xi_{1} t+\xi_{2} / t\right)} g(t) d t \text { for }\left(\xi_{1}, \xi_{2}\right) \in \mathbb{R}^{2},
$$

where $g(t)=f(t) \sqrt{1+1 / t^{4}}$. Let $\mathcal{F}$ be the subspace of all linear span of the functions $\left\{e_{n}^{p}(x)=\right.$ $\left.e^{\pi i(n+1 / p) x} ; n \in \mathbb{Z}\right\} \cup\left\{e_{n}^{\beta}(x)=e^{\pi i n \beta / x} ; n \in \mathbb{Z}\right\}$ in $L^{\infty}(\mathbb{R})$, where $p \in \mathbb{N}$ and $\beta$ is a positive real. By duality, Theorem 1.4 is equivalent to the following density result.

TheOrem 2.1. The set $\mathcal{F}$ is weak-star dense in $L^{\infty}(\mathbb{R})$ if and only if $0<\beta \leqslant p$.

\subsection{Proof of the necessary condition of Theorem 2.1}

Proof. The proof relies on the fact that bounded harmonic functions on the upper half-plane $\mathbb{C}_{+}=\{z \in \mathbb{C}: \operatorname{Im} z>0\}$ separate points of $\mathbb{C}_{+}$.

The functions $e_{n}^{p} ; n \in \mathbb{Z}$ and $e_{n}^{\beta} ; n \in \mathbb{Z}$ extend to bounded harmonic functions on $\mathbb{C}_{+}$, by letting

$$
e_{n}^{p}(z)=\left\{\begin{array}{l}
e^{\pi i(n+1 / p) z}, \operatorname{Im} z>0, n \geqslant 0 \\
e^{\pi i(n+1 / p) \bar{z}}, \operatorname{Im} z>0, n<0
\end{array}\right.
$$

and

$$
e_{n}^{\beta}(z)=\left\{\begin{array}{l}
e^{\pi i n \beta / \bar{z}}, \operatorname{Im} z>0, n \geqslant 0 \\
e^{\pi i n \beta / z}, \operatorname{Im} z>0, n<0
\end{array}\right.
$$


For $f \in L^{\infty}(\mathbb{R})$ the bounded harmonic function extension of $f$ on $\mathbb{C}_{+}$is defined via point evaluation with respect to the Poisson kernel $P_{z}$ :

$$
f(z)=\frac{1}{\pi} \int_{\mathbb{R}} f(t) P_{z}(t) d t
$$

where $z=x+i y \in \mathbb{C}_{+}$, and $P_{z}(t)=\frac{y}{(x-t)^{2}+y^{2}}, t \in \mathbb{R}$. As $P_{z}(\cdot) \in L^{1}(\mathbb{R})$, for $z \in \mathbb{C}_{+}$, the point evaluation $f \rightarrow f(z)$ defines a weak-star continuous functional on $L^{\infty}(\mathbb{R})$.

Moreover it is known that all bounded harmonic functions on $\mathbb{C}_{+}$arise as bounded harmonic extensions of functions in $L^{\infty}(\mathbb{R})$. Therefore, if $\mathcal{F}$ is weak-star dense in $L^{\infty}(\mathbb{R})$, then $\mathcal{F}$ must separate points of $\mathbb{C}_{+}$.

Solving for $z_{1}, z_{2} \in \mathbb{C}_{+}$such that

$$
\begin{aligned}
& e_{n}^{p}\left(z_{1}\right)=e_{n}^{p}\left(z_{2}\right) \\
& e_{n}^{\beta}\left(z_{1}\right)=e_{n}^{\beta}\left(z_{2}\right)
\end{aligned} \text { for all } n \in \mathbb{Z}
$$

we get

$$
\left\{\begin{array}{l}
z_{1}-z_{2} \in 2 p \mathbb{Z} \\
\frac{1}{z_{1}}-\frac{1}{z_{2}} \in \frac{2}{\beta} \mathbb{Z} .
\end{array}\right.
$$

In view of [12, Lemma 1.5], with $m=n=1, a=2 p$ and $b=2 / \beta$, we therefore have

$$
z_{1}=p(1+\mathrm{i} \sqrt{\beta / p-1}), \quad z_{2}=p(-1+\mathrm{i} \sqrt{\beta / p-1})
$$

which are two distinct points of $\mathbb{C}_{+}$if and only if $p<\beta$. As a consequence if $p<\beta, \mathcal{F}$ does not separate points of $\mathbb{C}_{+}$.

Thus the condition $0<\beta \leqslant p$ is necessary for $\left(\Gamma, \Lambda_{\beta}^{\theta}\right)$ to be a Heisenberg uniqueness pair.

\subsection{Proof of sufficient condition in Theorem 2.1}

To prove the sufficient condition in Theorem 2.1, we need the following interlude on Gauss-type maps and invariant measures. In what follows we assume that $0<\beta \leqslant p$.

A Gauss-type map. For $u \in \mathbb{R}$, the expression $\{u\}_{2}$ is the unique number in $(-1,1]$ such that $u-\{u\}_{2} \in 2 \mathbb{Z}$. We consider a Gauss-type map $\tau$ on the interval $(-p, p]$, which is topologically equivalent to $\mathbb{R} / 2 p \mathbb{Z}$. The map $\tau$ is defined by letting

$$
\tau(x)=\left\{\begin{array}{cc}
\left\{-\frac{p}{x}\right\}_{2}, & x \neq 0 \\
0 & x=0 .
\end{array}\right.
$$

Observe that, for $j \in \mathbb{Z}^{*}=\mathbb{Z} \backslash\{0\}, \tau$ can be expressed as

$$
\tau(x)=-\frac{p}{x}+2 j \text { whenever } \frac{p}{2 j+1}<x \leqslant \frac{p}{2 j-1},
$$

and hence $\tau:\left(\frac{p}{2 j+1}, \frac{p}{2 j-1}\right] \rightarrow(-1,1]$ is one-to-one and for $x \in(-p, p] \backslash \frac{p}{2 \mathbb{Z}+1}$, the derivative of $\tau$ is $\tau^{\prime}(x)=\frac{p}{x^{2}}$. Next, we define the map $U:(-p, p] \rightarrow(-p, p]$ by letting

$$
U(x)=p \tau(x) .
$$

Invariant measures for Gauss-type map. If $\varphi$ is a continuous $2 p$-periodic function on $\mathbb{R}$ and $\nu$ is a finite complex-valued Borel measure on $(-p, p]$, then the integral

$$
\int_{(-p, p]} \varphi(x) d \nu(x)
$$

is well defined. A function $\varphi$ on $(-p, p]$ is said to be pseudo-continuous, if for every open subset $\mathcal{O}$ of $(-p, p]$ such that $(-p, p] \backslash \mathcal{O}$ is countable, $\varphi$ is bounded on $(-p, p]$ and continuous on $\mathcal{O}$. 
In fact, (2.3) makes sense if $\varphi$ is pseudo-continuous. Also observe that for a pseudo-continuous function $\varphi$ on $(-p, p], \varphi \circ U$ is pseudo-continuous.

A finite complex Borel measure $\nu$ on $(-p, p]$ is $U$-invariant provided that

$$
\int_{(-p, p]} \varphi(U(x)) d \nu(x)=\int_{(-p, p]} \varphi(x) d \nu(x)
$$

holds for all pseudo-continuous functions $\varphi$. For details about invariant measures for continuous maps see [6, p. 97]. Rewriting the left-hand side of (2.4), we get

$$
\begin{aligned}
\int_{(-p, p] \backslash\{0\}} \varphi(U(x)) d \nu(x) & =\sum_{j \in \mathbb{Z}^{*}} \int_{\left(\frac{p}{2 j+1}, \frac{p}{2 j-1}\right]} \varphi(p \tau(x)) d \nu(x) \\
& =\sum_{j \in \mathbb{Z}^{*}} \int_{\left(\frac{p}{2 j+1}, \frac{p}{2 j-1}\right]} \varphi\left(p\left(-\frac{p}{x}+2 j\right)\right) d \nu(x) \\
& =\sum_{j \in \mathbb{Z}^{*}} \int_{(-p, p]} \varphi(t) d \nu\left(\frac{p^{2}}{2 p j-t}\right) \\
& =\sum_{j \in \mathbb{Z}^{*}} \int_{(-p, p]} \varphi(t) d \nu_{j}(t),
\end{aligned}
$$

where $d \nu_{j}(t)=d \nu\left(\frac{p^{2}}{2 p j-t}\right)$. Thus it follows that $\nu$ is $U$-invariant provided that

$$
\nu=\nu(0) \delta_{0}+\sum_{j \in \mathbb{Z}^{*}} \nu_{j}
$$

In addition, given $\lambda \in \mathbb{C}$, complex Borel measure $\nu$ on $(-p, p]$ is $(U, \lambda)$-invariant provided that

$$
\int_{(-p, p]} \varphi(U(x)) d \nu(x)=\lambda \int_{(-p, p]} \varphi(x) d \nu(x)
$$

holds for all pseudo-continuous functions $\varphi$. Equivalently $\nu$ is $(U, \lambda)$-invariant if

$$
\lambda \nu=\nu(0) \delta_{0}+\sum_{j \in \mathbb{Z}^{*}} \nu_{j}
$$

It is easy to see that, for $|\lambda|>1$, there are no $(U, \lambda)$-invariant measures except for the zero measure.

Invariant measure of infinite mass. Consider the positive $\sigma$-finite measure $\omega$ on $(-p, p]$ of infinite mass by letting

$$
d \omega(x)=\frac{d x}{p^{2}-x^{2}} .
$$

Then the measure $\omega$ is $U$-invariant as

$$
d \omega_{j}(t)=d \omega\left(\frac{p^{2}}{2 p j-t}\right)=\frac{d t}{(2 p j-t)^{2}-p^{2}} \text { and } \sum_{j \in \mathbb{Z}^{*}} \frac{1}{(2 p j-t)^{2}-p^{2}}=\frac{1}{p^{2}-t^{2}},
$$

and therefore $\omega$ satisfies

$$
\omega=\omega(0) \delta_{0}+\sum_{j \in \mathbb{Z}^{*}} \omega_{j}
$$

Let $|U|:[0, p] \rightarrow[0, p]$ be the map $|U|(x)=|U(x)|$. It can be shown that the measure $\omega$ is $|U|$-invariant. Also $|U|$ is ergodic. As a consequence of the Birkhoff ergodic theorem we get the following result. 
Proposition 2.2. Suppose $\lambda \in \mathbb{C}$ with $|\lambda|=1$. Let $\nu$ be a absolutely continuous finite complex $(U, \lambda)$-invariant Borel measure on $(-p, p]$. Then $\nu$ is zero.

The proof of Proposition 2.2 works along the same lines as in proof of [12, Proposition 2.4].

Gauss-type map for $0<\beta<p$. The Gauss-type map $\tau_{\beta}$ is defined by $\tau_{\beta}(0)=0$, and for $x \neq 0$,

$$
\tau_{\beta}(x)=\left\{-\frac{\beta}{x}\right\}_{2}
$$

and the map $U_{\beta}:(-p, p] \rightarrow(-p, p]$ which is associated to $\tau_{\beta}$ is defined by letting

$$
U_{\beta}(x)=p \tau_{\beta}(x) .
$$

Proposition 2.3. For $\lambda \in \mathbb{C}$ with $|\lambda|=1$, a finite complex Borel measure $\nu$ on $(-p, p]$ is $\left(U_{\beta}, \lambda\right)$-invariant provided that

$$
\lambda \nu=\nu(0) \delta_{0}+\sum_{j \in \mathbb{Z}^{*}} \nu_{j}
$$

where $d \nu_{j}(t)=d \nu\left(\frac{p \beta}{2 p j-t}\right), t \in(-p, p]$.

Proof. A finite complex Borel measure $\nu$ on $(-p, p]$ is $\left(U_{\beta}, \lambda\right)$-invariant provided that

$$
\int_{(-p, p]} \varphi\left(U_{\beta}(x)\right) d \nu(x)=\lambda \int_{(-p, p]} \varphi(x) d \nu(x)
$$

holds for all pseudo-continuous test functions $\varphi$ on $(-p, p]$. Observe that

$$
\begin{aligned}
\int_{(-p, p] \backslash\{0\}} \varphi\left(U_{\beta}(x)\right) d \nu(x) & =\sum_{j \in \mathbb{Z}^{*}} \int_{\left(\frac{\beta}{2 j+1}, \frac{\beta}{2 j-1}\right]} \varphi\left(p \tau_{\beta}(x)\right) d \nu(x) \\
& =\sum_{j \in \mathbb{Z}^{*}} \int_{\left(\frac{\beta}{2 j+1}, \frac{\beta}{2 j-1}\right]} \varphi\left(p\left(-\frac{\beta}{x}+2 j\right)\right) d \nu(x) \\
& =\sum_{j \in \mathbb{Z}^{*}} \int_{(-p, p]} \varphi(t) d \nu\left(\frac{p \beta}{2 p j-t}\right) \\
& =\sum_{j \in \mathbb{Z}^{*}} \int_{(-p, p]} \varphi(t) d \nu_{j}(t) .
\end{aligned}
$$

Thus a measure $\nu$ is $\left(U_{\beta}, \lambda\right)$-invariant only if

$$
\lambda \nu=\nu(0) \delta_{0}+\sum_{j \in \mathbb{Z}^{*}} \nu_{j}
$$

The sum space. Let $L_{p}^{\infty}(\mathbb{R})$ denote the subspace of all functions $f \in L^{\infty}(\mathbb{R})$ such that the map $x \longmapsto e^{-\pi i x / p} f(x)$ is 2-periodic. Then the weak-star closure in $L^{\infty}(\mathbb{R})$ of linear span of $\left\{e_{n}^{p}(x)=e^{\pi i(n+1 / p) x} ; n \in \mathbb{Z}\right\}$ equals $L_{p}^{\infty}(\mathbb{R})$.

Similarly, let $L_{\beta}^{\infty}(\mathbb{R})$ denote the subspace of all functions $f \in L^{\infty}(\mathbb{R})$ such that the map $x \longmapsto f(\beta / x)$ is 2-periodic. Then the weak-star closure in $L^{\infty}(\mathbb{R})$ of linear span of $\left\{e_{n}^{\beta}(x)=\right.$ $e^{\pi i n \beta / x} ; n \in \mathbb{Z}$ \} equals $L_{\beta}^{\infty}(\mathbb{R})$.

Consider the sum space $L_{p}^{\infty}(\mathbb{R})+L_{\beta}^{\infty}(\mathbb{R})$. The sufficient part of Theorem 2.1 is equivalent to the following density result. 
TheOREM 2.4. For $0<\beta \leqslant p$, the sum space

$$
L_{p}^{\infty}(\mathbb{R})+L_{\beta}^{\infty}(\mathbb{R})
$$

is weak-star dense in $L^{\infty}(\mathbb{R})$.

The composition operator. Observe that the functions in $L_{p}^{\infty}(\mathbb{R})$ are defined freely on $(-p, p]$, and because of periodicity they are uniquely determined on $\mathbb{R} \backslash(-p, p]$. Similarly, the functions in $L_{\beta}^{\infty}(\mathbb{R})$ are defined freely on $\mathbb{R} \backslash(-\beta, \beta]$, and because of periodicity they are uniquely determined on $(-\beta, \beta]$. For $E \subseteq \mathbb{R}$, the function $\chi_{E}$ denotes the characteristic function of $E$ on $\mathbb{R}$.

Define operator $\mathrm{S}: L^{\infty}((-p, p]) \rightarrow L^{\infty}(\mathbb{R} \backslash(-p, p])$ by

$$
\mathrm{S}[\varphi](x)=\varphi\left(p\{x / p\}_{2}\right) \chi_{\mathbb{R} \backslash(-p, p]}(x),
$$

where $\varphi \in L^{\infty}((-p, p])$ and $x \in \mathbb{R}$.

The operator $\mathrm{T}_{\beta}: L^{\infty}(\mathbb{R} \backslash(-\beta, \beta]) \rightarrow L^{\infty}((-\beta, \beta])$ is defined by

$$
\mathrm{T}_{\beta}[\psi](x)=\psi\left(\frac{\beta}{\{\beta / x\}_{2}}\right) \chi_{(-\beta, \beta]}(x),
$$

where $\psi \in L^{\infty}(\mathbb{R} \backslash(-\beta, \beta])$ and $x \in \mathbb{R}$. A simple computation shows that for $\psi \in L^{\infty}(\mathbb{R} \backslash$ $(-\beta, \beta])$,

$$
\mathrm{S}\left[\psi \circ I_{\beta}\right] \circ I_{\beta}=\mathrm{T}_{\beta}[\psi]
$$

where $I_{\beta}(x)=-\beta / x$. Then the composition operator $\mathrm{T}_{\beta} \mathrm{S}: L^{\infty}((-p, p]) \rightarrow L^{\infty}((-p, p])$ is defined as

$$
\mathrm{T}_{\beta} \mathrm{S}[\varphi](x)=\varphi\left(p\left\{\frac{\beta_{0}}{\{\beta / x\}_{2}}\right\}_{2}\right) \chi_{E_{\beta}}(x),
$$

for $\varphi \in L^{\infty}((-p, p])$, where $\beta_{0}=\beta / p$ and $E_{\beta}=\left\{x \in(-\beta, \beta] \backslash\{0\}: \frac{\beta_{0}}{\{\beta / x\}_{2}} \in \mathbb{R} \backslash(-1,1]\right\}$.

The Koopman operator. For $0<\beta \leqslant p$, consider the weighted Koopman operator $\mathcal{C}_{\beta}: L^{\infty}((-p, p]) \rightarrow L^{\infty}((-p, p])$ associated to $U_{\beta}$ by

$$
\mathcal{C}_{\beta}[\varphi](x)=\varphi \circ U_{\beta}(x) \chi_{(-\beta, \beta]}(x),
$$

where $x \in \mathbb{R}$. The predual adjoint $\mathcal{C}_{\beta}^{*}$ of $\mathcal{C}_{\beta}$ is the Perron-Frobenius operator $\mathcal{P}_{\beta}: L^{1}((-p, p]) \rightarrow$ $L^{1}((-p, p])$ given by

$$
\mathcal{P}_{\beta}[f](x)=\sum_{j \in \mathbb{Z}^{*}} \frac{p \beta}{(2 p j-x)^{2}} f\left(\frac{p \beta}{2 p j-x}\right) .
$$

The operator $\mathcal{P}_{\beta}$ is linear and a norm contraction, that is,

$$
\left\|\mathcal{P}_{\beta}[f]\right\|_{L^{1}((-p, p])} \leqslant\|f\|_{L^{1}((-p, p])},
$$

for $f \in L^{1}((-p, p])$. Thus the point spectrum $\sigma_{\text {point }}\left(\mathcal{P}_{\beta}\right)$ of $\mathcal{P}_{\beta}$ is contained in the closed unit $\operatorname{disk} \overline{\mathbb{D}}=\{\lambda \in \mathbb{C}:|\lambda| \leqslant 1\}$.

A simple computation gives $\mathrm{T}_{\beta} \mathrm{S}=\mathcal{C}_{\beta}^{2}$. Thus the operator $\mathrm{I}-\mathrm{T}_{\beta} \mathrm{S}: L^{\infty}((-p, p]) \rightarrow$ $L^{\infty}((-p, p])$ can be expressed as

$$
\mathrm{I}-\mathrm{T}_{\beta} \mathrm{S}=\left(\mathrm{I}+\mathcal{C}_{\beta}\right)\left(\mathrm{I}-\mathcal{C}_{\beta}\right) .
$$

Proposition 2.5. The range of the operator $I-T_{\beta} S$ is weak-star dense in $L^{\infty}((-p, p])$. 
Proof. In view of the identity (2.6), we prove that the operators $\mathrm{I}+\mathcal{C}_{\beta}$ and $\mathrm{I}-\mathcal{C}_{\beta}$ have weak-star dense range. For $\lambda= \pm 1$, the weak-star closure of the range of $\lambda \mathrm{I}-\mathcal{C}_{\beta}$ equals to $L^{\infty}((-p, p])$ if and only if the predual adjoint

$$
\lambda \mathrm{I}-\mathcal{P}_{\beta}: L^{1}((-p, p]) \rightarrow L^{1}((-p, p])
$$

has null kernel. Therefore, it remains to show that \pm 1 are not eigenvalues of $\mathcal{P}_{\beta}$.

We actually prove a stronger statement, namely that if $\lambda \in \mathbb{C}$ with $|\lambda|=1$, then $\lambda$ is not an eigenvalue of $\mathcal{P}_{\beta}$.

Case 1. Assume that $\beta=p$ and recall that $\mathcal{C}_{p}[\varphi](x)=\varphi \circ U(x) \chi_{(-p, p]}(x)$. By the dual relation, we get

$$
\int_{(-p, p]} \varphi(x) \mathcal{P}_{p}[\psi](x) d x=\int_{(-p, p]} \varphi \circ U(x) \psi(x) d x .
$$

Suppose $\psi_{0}$ is a non-zero eigenfunction of $\mathcal{P}_{p}$ with eigenvalue $\lambda$ such that $|\lambda|=1$, then $\mathcal{P}_{p}\left[\psi_{0}\right]=$ $\lambda \psi_{0}$ and therefore

$$
\lambda \int_{(-p, p]} \varphi(x) \psi_{0}(x) d x=\int_{(-p, p]} \varphi(U(x)) \psi_{0}(x) d x .
$$

Hence a nonzero absolutely continuous finite Borel measure $d \nu(x)=\psi_{0}(x) d x$ is $(U, \lambda)$-invariant which contradicts Proposition 2.2. Thus $|\lambda|=1$ is not an eigenvalue of $\mathcal{P}_{p}$.

Case 2. Assume $0<\beta<p$. By dual relation, we get

$$
\lambda \int_{(-p, p]} \varphi(x) d \nu(x)=\int_{(-\beta, \beta]} \varphi\left(p \tau_{\beta}(x)\right) d \nu(x)
$$

for all $\varphi \in L^{\infty}((-p, p])$, where $\lambda \in \mathbb{C}$ with $|\lambda|=1$ and $\nu$ is a absolutely continuous finite Borel measure on $(-p, p]$. It follows that

$$
\lambda d \nu(x)=\sum_{j \in \mathbb{Z}^{*}} d \nu_{j}(x), \quad x \in(-p, p],
$$

where $d \nu_{j}(x)=d \nu\left(\frac{\beta p}{2 p j-x}\right)$. Taking absolute values and then integrating over $(-p, p]$, we have

$$
\int_{(-p, p]}|d \nu(x)| \leqslant \sum_{j \in \mathbb{Z}^{*}} \int_{(-p, p]}\left|d \nu_{j}(x)\right|=\int_{(-\beta, \beta]}|d \nu(x)|, \quad x \in(-p, p],
$$

which is only possible if the following holds:

$$
|d \nu(x)|=\sum_{j \in \mathbb{Z}^{*}}\left|d \nu_{j}(x)\right|, \quad x \in(-p, p] .
$$

From the above equality, it follows that

$$
d \nu(x)=0 \text { for } x \in(-p, p] \backslash(-\beta, \beta] .
$$

Iterating the equation (2.7) and using the above arguments repeatedly, we conclude that

$$
d \nu(x)=0 \text { for } x \in(-p, p] \backslash E_{\beta}(n),
$$

where $E_{\beta}(n)=\left\{x \in(-p, p]: U_{\beta}^{(k)}(x) \in(-\beta, \beta]\right.$ for $\left.k=0, \ldots, n-1\right\}$ and $U_{\beta}^{(k)}$ the $k$ th iteration of $U_{\beta}$. By letting $n \rightarrow+\infty$, we get that

$$
d \nu(x)=0 \text { for } x \in(-p, p] \backslash E_{\beta}(\infty)
$$

with

$$
E_{\beta}(\infty)=\left\{x \in(-p, p]: U_{\beta}^{(k)}(x) \in(-\beta, \beta] \text { for } k=0,1,2, \ldots\right\}
$$


The set $E_{\beta}(\infty)$ is $U_{\beta}$ invariant and it has zero length. Hence the absolutely continuous measure $\nu$ vanishes almost everywhere on $(-p, p]$. Thus we conclude that $|\lambda|=1$ is not an eigenvalue of $\mathcal{P}_{\beta}$.

Finally, we show that for $0<\beta \leqslant p$, the sum space is weak-star dense in $L^{\infty}(\mathbb{R})$.

Proof of Theorem 2.4. The proof is similar to the proof of [12, Lemma 5.2], hence omitted. This completes the proof of Theorem 2.4.

Acknowledgements. The authors would like to thank the referee for suggestions that helped in improving the presentation. The first named author gratefully acknowledges the support provided by IIT Kanpur, Government of India.

\section{References}

1. D. B. Вавот, 'Heisenberg uniqueness pairs in the plane, three parallel lines', Proc. Amer. Math. Soc. 141 (2013) 3899-3904.

2. S. BAGCHI, 'Heisenberg uniqueness pairs corresponding to a finite number of parallel lines', Adv. Math. 325 (2018) 814-823.

3. M. Benedicks, 'On Fourier transforms of functions supported on sets of finite Lebesgue measure', J. Math. Anal. Appl. 106 (1985) 180-183.

4. F. Canto-Martín, H. Hedenmalm and A. Montes-Rodríguez, 'Perron-Frobenius operators and the Klein-Gordon equation', J. Eur. Math. Soc. 16 (2014) 31-66.

5. A. Chattopadhyay, S. Ghosh, D. K. Giri and R. K. Srivastava, 'Heisenberg uniqueness pairs on the Euclidean spaces and the motion group', C. R. Math. Acad. Sci. Paris 358 (2020) 365-377.

6. M. EinsiedLeR and T. WARD, Ergodic theory with a view towards number theory, Graduate Texts in Mathematics 259 (Springer, London, 2011). MR2723325.

7. D. K. GiRI and R. K. Srivastava, 'Heisenberg uniqueness pairs for some algebraic curves in the plane', Adv. Math. 310 (2017) 993-1016.

8. D. K. GiRI and R. K. SRIVAstava, 'Heisenberg uniqueness pairs for the finitely many parallel lines with an irregular gap', Preprint, 2017, arXiv:1605.06724v6.

9. F. J. Gonzaléz Vieli, 'A uniqueness result for the Fourier transform of measures on the sphere', Bull. Aust. Math. Soc. 86 (2012) 78-82.

10. K. Gröchenig and P. Jaming, 'The Cramér-Wold theorem on quadratic surfaces and Heisenberg uniqueness pairs', J. Inst. Math. Jussieu 19 (2020) 117-135.

11. V. HAVIN and B. JÖRICKE, The uncertainty principle in harmonic analysis, Ergebnisse der Mathematik und ihrer Grenzgebiete (3) [Results in Mathematics and Related Areas (3)] 28 (Springer, Berlin, 1994).

12. H. Hedenmalm and A. Montes-Rodríguez, 'Heisenberg uniqueness pairs and the Klein-Gordon equation', Ann. of Math. (2) 173 (2011) 1507-1527.

13. H. Hedenmalm and A. Montes-Rodríguez, 'The Klein-Gordon equation, the Hilbert transform, and dynamics of Gauss-type maps', J. Eur. Math. Soc. 22 (2020) 1703-1757.

14. H. Hedenmalm and A. Montes-Rodríguez, 'The Klein-Gordon equation, the Hilbert transform, and Gauss-type maps: $H^{\infty}$ approximation', J. Anal. Math., to appear.

15. W. Heisenberg, 'Über den anschaulichen Inhalt der quantentheoretischen Kinematik und Mechanik', $Z$. Physik 43 (1927) 172-198.

16. P. Jaming and K. Kellay, 'A dynamical system approach to Heisenberg uniqueness pairs', J. Anal. Math. 134 (2018) 273-301.

17. N. Lev, 'Uniqueness theorem for Fourier transform', Bull. Sci. Math. 135 (2011) 134-140.

18. P. SJÖLIn, 'Heisenberg uniqueness pairs and a theorem of Beurling and Malliavin', Bull. Sci. Math. 135 (2011) $125-133$

19. P. SJÖLIn, 'Heisenberg uniqueness pairs for the parabola', J. Fourier Anal. Appl. 19 (2013) 410-416.

20. R. K. Srivastava, 'Non-harmonic cones are Heisenberg uniqueness pairs for the Fourier transform on $\mathbb{R}^{n}$, J. Fourier Anal. Appl. 24 (2018) 1425-1437. 
Deb Kumar Giri

Department of Mathematics Indian Institute of Technology

Kanpur 208016

India

Current address:

Department of Mathematics

Indian Institute of Science

Bangalore 560012

India

dgiri@iitk.ac.in debkumarg@iisc.ac.in
Rama Rawat

Department of Mathematics

Indian Institute of Technology

Kanpur 208016

India

rrawat@iitk.ac.in

The Bulletin of the London Mathematical Society is wholly owned and managed by the London Mathematical Society, a not-for-profit Charity registered with the UK Charity Commission. All surplus income from its publishing programme is used to support mathematicians and mathematics research in the form of research grants, conference grants, prizes, initiatives for early career researchers and the promotion of mathematics. 